\title{
Sub-band Beamforming of OFDM Signals in Time Varying
}

\section{Multi-Path fading Channel}

\author{
Jahangir Dadkhah Chimeh $^{1}$, Hamid Reza Bakhshi ${ }^{2}$, Mohammad Bagher Menhaj ${ }^{3}$ \\ ${ }^{1}$ Islamic Azad University, Saveh Branch, Saveh, Iran \\ 2 \\ Shahed University, Tehran, Iran, Corresponding Author \\ 3 AmirKabir University Of Technology, Tehran, Iran \\ Dadkhah@itrc.ac.ir, Karami@itrc.ac.ir, hamidreza_bakhshi@yahoo.com
}

\begin{abstract}
In this paper, a semi-blind beamforming algorithm is proposed for Orthogonal Frequency Division Multiplexing (OFDM) signals to combat multipath fading which is based on semi-blind beamforming of each sub-band in a OFDM system using Decision Directed Algorithm (DDA). The proposed algorithm is simulated for time varying channel with Clark model for block to block variation of the channel coefficients it is shown that without semi-blind operation error floor occurs in the performance of the sub-band beamformer but in the blind mode of the operation, by exploiting the information contents in the decisions the sub-band beamformer provides efficient bit error rate versus $E_{b} / N_{0}$ even in different block length and modulation schemes.
\end{abstract}

Index Terms- OFDM, Sub-band beamforming, Doppler frequency, semi-blind beamforming.

\section{Introduction}

Recently demand for high speed data rate services necessitates the use of wideband channels. But in this case, frequency selective fading is appeared, i.e. a large number of resolvable multi-paths components affect the performance of the system by introducing Inter-Symbol-Interference (ISI). OFDM is well-known to be effective against multipath distortion. With OFDM signaling a wideband channel converts to many narrowband flat sub-channels [1]. Deployment of the antenna arrays, not only provide SNR gain and improves spectral efficiency but also offers diversity gain. Although there are many research activities in OFDM, only a few numbers of them involve in adaptive beamforming of OFDM signals. When OFDM signaling is used, each sub-channel tolerates different fading factor and therefore for each sub-channel a separate beamformer is required [2], [3]. In this paper, semiblind decision directed algorithm is proposed to beamforming of OFDM signals where first part of each stream is devoted to training symbols and other part is data signals. In the blind mode of the operation the estimated data symbols is used as virtual training symbols.

The rest of this paper is organized as follows. In Section II, the system model for OFDM signals and time variation of the channel is presented. In Section III, Simulation results of the proposed sub-band beam-former is presented and is compared to the conventional training based sub-band beamformer. Paper is concluded is Section IV.

\section{System Model}

Consider $d_{0}, d_{1}, d_{2}, \ldots, d_{N-1}$ as a stream of data, where $d_{i}$ s are complex symbols which his produced by a digital modulator such as PSK or QAM. After applying IFFT transform on this stream OFDM symbols are generated as follows.

$$
s_{k}=\sum_{n=0}^{N-1} d_{n} \exp \left(j 2 \pi \frac{n k}{N}\right)=\sum_{n=0}^{N-1} d_{n} \exp \left(j 2 \pi f_{n} t_{k}\right)
$$

Where $t_{k}=k T_{s}, f_{n}=\frac{n}{N T_{s}}, n=0,1, . ., N-1$ and $T_{s}$ is the period of signal. This stream is transmitted through a time varying multi-path fading channel and is received by $M$ elements of the receiver array, as follows.

$$
r_{k}^{i}=\sum_{m=0}^{P} h_{m, k}^{i} s_{k-m}+w_{k}^{i}
$$

where $s_{k-m}$ is the transmitted symbol in time index, $k-m, w_{k}^{i}$ is the additive white Gaussian noise in the $i$ th received element, and $h_{m, k}^{i}$ is the $m$ th tap of the impulse response of order $P$ between transmitter and the $i$ th receiver. Therefore, in each time instance $N(P+1)$ channel parameters must be estimated. Each of these channel parameters is a combination of a constant part and a time varying part as

$$
h_{m, k}^{i}=h_{m, 0}^{i}+\tilde{h}_{m, k}^{i}
$$

where $h_{m, 0}^{i}$ is the nearly constant part which can be due to line of sight $(\mathrm{LoS})$ components that are fairly constant in a long block of data, and $\widetilde{h}_{m, k}^{i}$ is the time varying part which is due to non-LoS (NLoS) components that is severely variable 
in the duration of data block transmission with the autocorrelation that follows the following equation [4]

$$
E\left\{\tilde{h}_{m, k}^{i, j}\left[\widetilde{h}_{m, l}^{i, j}\right]^{*}\right\} \cong J_{0}\left(2 \pi f_{D}^{i, m} T|k-l|\right)
$$

where, $J_{0}($.$) is the zero-order Bessel function of the first kind,$ superscript $*$ denotes the complex conjugate, $f_{D}^{i, m}$ is Doppler frequency shift for $m$ th path between transmitter and $i$ th receiver, and $\mathrm{T}$ is the duration of each symbol. According to the wide sense stationary uncorrelated scattering (WSSUS) model of Bello [5], all the channel taps are independent, namely, all $h_{m, k}^{i} \mathrm{~s}$ vary independently, according to the autocorrelation model of (4). The normalized spectrum for each tap $\tilde{h}_{m, k}^{i}$ is

$$
S_{k}(f)= \begin{cases}\frac{1}{\pi f_{D}^{i, m} T \sqrt{1-\left(\frac{f}{f_{D}^{i, m}}\right)^{2}}}, & |f|<f_{D}^{i, m} T \\ 0, & \text { otherwise }\end{cases}
$$

The exact modeling of the vector process $\widetilde{h}_{m, k}^{i}$ with a finite length auto-regressive (AR) model is impossible. For implementation of a channel estimator, MIMO channel variations $\tilde{h}_{m, k}^{i}$ can be approximated by the following AR process of order $L$

$$
\tilde{h}_{m, k}^{i}=\sum_{l=1}^{L} \alpha_{i, m, l} \tilde{h}_{m, k-l}^{i}+v_{i, m, k}
$$

where $\alpha_{i, m, l}$ is $l$ th coefficient of $m$ th path from transmitter to $i$ th receiver and $v_{i, m, k} \mathrm{~s}$ are zero-mean i.i.d. complex Gaussian processes with variances given by

$$
E\left(v_{i, m, k}\left[v_{i, m, k}\right]^{*}\right)=\sigma_{v_{i, m, k}}^{2}
$$

For optimum selection of channel AR model parameters from correlation functions, Wiener equations can be used as solving the $L$ following equations

$$
\begin{aligned}
& J_{0}\left(2 \pi f_{D}^{i, m} T|k-t|\right)=\sum_{l=1}^{L} J_{0}\left(2 \pi f_{D}^{i, m} T|k-l-t|\right) \alpha_{i, m, l}, \\
& t=k-L, k-L+1, \ldots, k-1
\end{aligned}
$$

The length of the channel model must be chosen to a minimum of $90 \%$ of the energy spectrum of each channel coefficients which is contained in the frequency range of $|f|<f_{D}^{i, m} T$.

\section{Simulation Results}

The block diagram of this system is shown in Fig. 1. This system is simulated for 4 and 8PSK signaling and 32 and 64 sub-carrier OFDM systems. Three Rayleigh paths propagation is assumed and the variances of these three paths are assumed to be $1,0.8$, and 0.6 respectively. The carrier frequency and symbol rate are assumed to be $1.8 \mathrm{GHz}$ and $2 \mathrm{Mbps}$ respectively. Also the speed of mobile users is assumed to be $200 \mathrm{Mph}$ which is a relatively high speed for vehicles. In all simulation first 5 blocks are considered as training data and 45 next blocks are assigned to data symbols. The performance of semi-blind algorithm is compared to conventional sub-band beamformer and its results is shown in Figs. 2-4. Figs. 2 and 3 present the simulation results for 4PSK and 8PSK signaling respectively while number of sub-carriers is 32 , and Figs. 4 and 5 present the same simulations for 64 sub-carrier case. As it can be seen form these Figures, in all cases, conventional sub-band beamformer encounters to error floor and therefore by increasing the $E_{b} / N_{0}$ bit error rate is remained unchanged. This phenomenon is due to failing the conventional training based sub-band beamformer. But by exploiting information contents in detected signals in the blind mode of the operation the level of error floor is much decreased and for 8PSK signaling error floor is disappeared.

\section{Concluding Remarks}

In this paper semi-blind sub-band beamformer is proposed for OFDM signals. Decision directed algorithm is used to operate in the blind mode when the transmitted data is unknown. The proposed algorithm is simulated for 4PSK and 8PSK signaling, and 32 and 64 sub-carrier cases. The speed of user is assumed to be $200 \mathrm{Mph}$ and the first 5 blocks in each 50 transmitted blocks are assigned to training. System is simulated by Monte Carlo simulation technique and it is shown that the performance of the conventional sub-band beamformer encounters to error floor in this channel variation speed. But the proposed semi-blind beamformer provides error floor in much lower levels especially for 8PSK signaling.

\section{References}

[1] R. V. Nee, and R. Prasad, OFDM for Wireless Multimedia Communications, Boston, Artech House, 1998.

[2] B. L. P. Cheung, F. Alam, J. H. Reed, and B. D. Woerner, "New Adaptive Beamforming Algorithms for OFDM Systems," Proceedings of the $14^{\text {th }}$ Annual International Conference on Wireless Communications, July 2002, Calgary, Alberta, Canada.

[3] F. Alam, Space Time Processing for Third Generation CDMA Systems, Ph. D. Dissertation, Virginia Tech, Nov. 2003.

[4] W. C. Jakes Jr., Microwave Mobile Communications. John Wiley \& Sons, NY, 1974.

[5] P. A. Bello, "Characterization of randomly time-variant linear channels," Trans. On Communication Systems, vol. CS-11, pp. 360-393, Dec. 1963. 


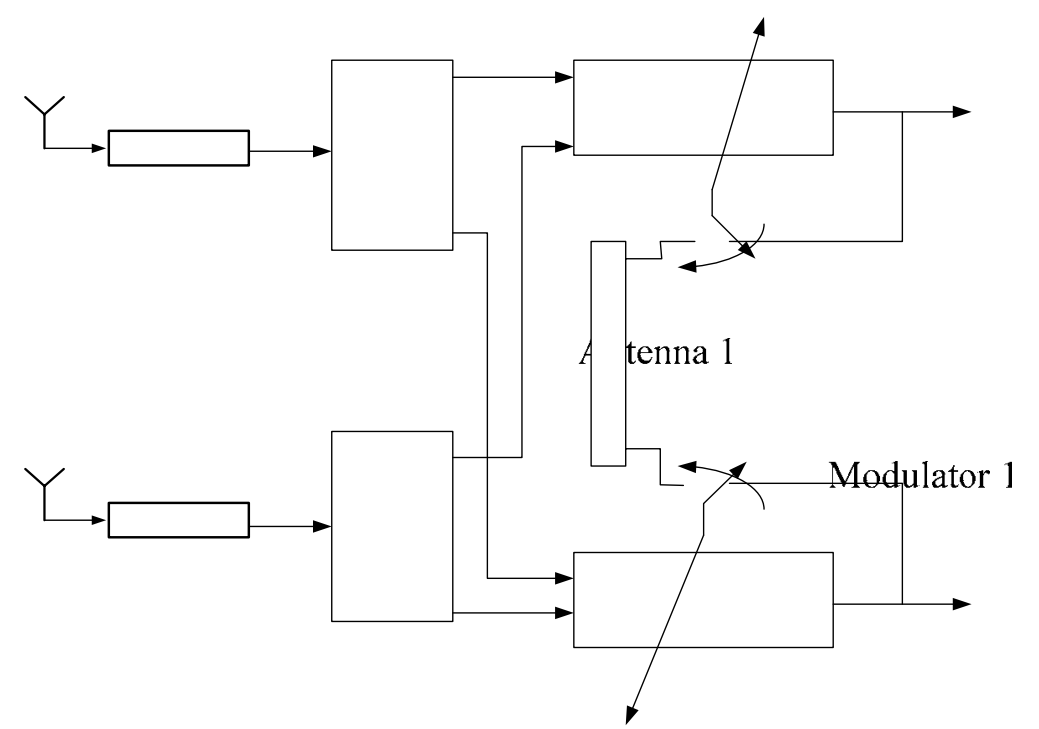

Fig. 1. Block diagram of the semi-blind sub-band beamformer

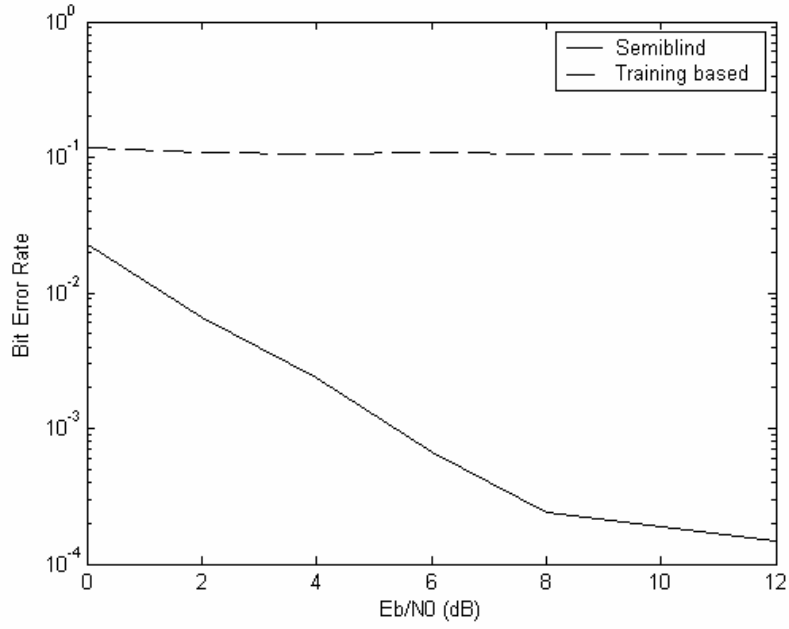

Fig. 2. Performance comparison of semi-blind sub-band beamformer for 32 symbols blocks and 4PSK signaling

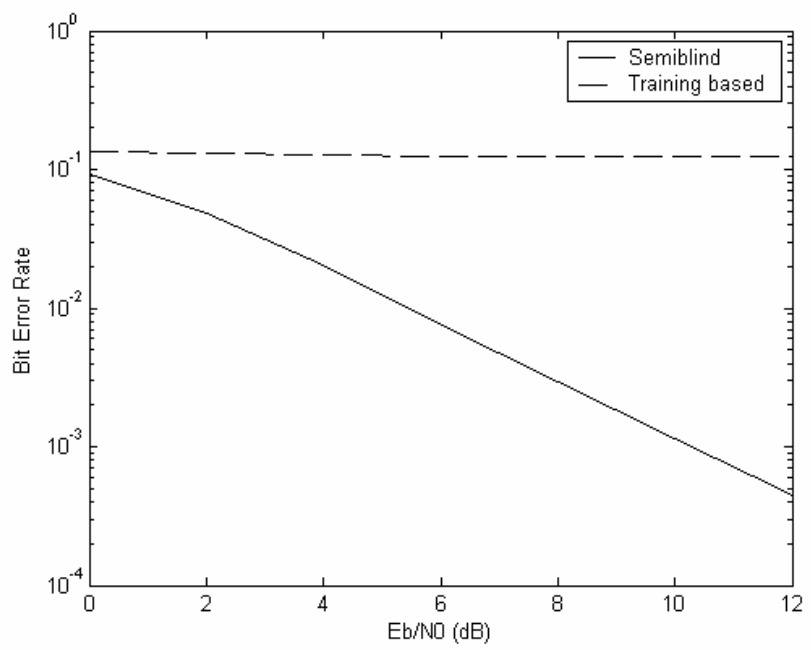

Fig. 3. Performance comparison of semi-blind sub-band beamformer for 32 symbols blocks and 8PSK signaling

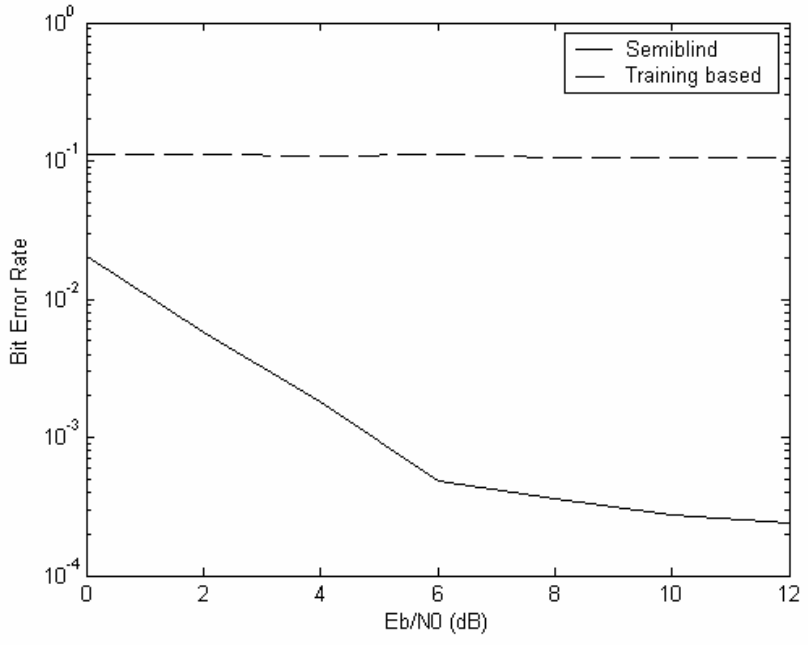

Fig. 4. Performance comparison of semi-blind sub-band beamformer for 64 symbols blocks and 4PSK signaling

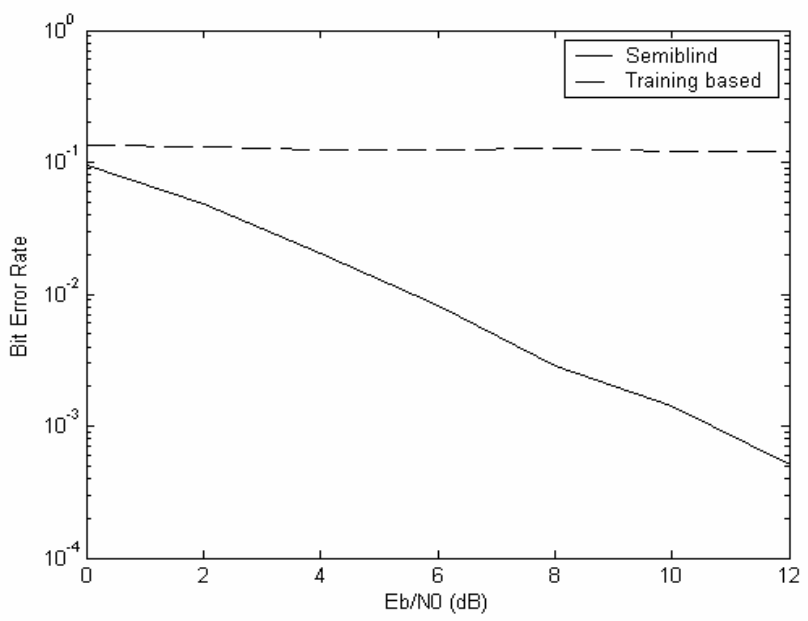

Fig. 5. Performance comparison of semi-blind sub-band beamformer for 64 symbols blocks and 8PSK signaling 